\title{
High C-reactive protein/albumin ratio predicts unfavorable distant metastasis-free survival in nasopharyngeal carcinoma: a propensity score-matched analysis
}

This article was published in the following Dove Press journal:

Cancer Management and Research

\author{
Yan Wang ${ }^{1-3, *}$ \\ Lin Yang ${ }^{1-3, *}$ \\ Liangping $\mathrm{Xia}^{1-3}$ \\ Yong Chen ${ }^{1-3}$
}

'Department of VIP, Sun Yat-sen University Cancer Center, Guangzhou, People's Republic of China; ${ }^{2}$ State Key Laboratory of Oncology in Southern China, Guangzhou, People's Republic of China; ${ }^{3}$ Collaborative Innovation Center for Cancer Medicine, Guangzhou, People's Republic of China

*These authors contributed equally to this work
Correspondence: Liangping Xia; Yong Chen

Sun Yat-sen University Cancer Center, 65I Dongfeng Road East, Guangzhou 510060, People's Republic of China Tel +862087343107; +862087343505 Emailxialp@sysucc.org.cn; chenyong@mail.sysu.edu.cn
Background: Recent studies have indicated that the $\mathrm{C}$-reactive protein/albumin (CRP/ALB) ratio (CAR) may represent a simple inflammation-based index for assessing the host inflammatory response. In this study, the prognostic value of the CAR for distant metastasis-free survival (DMFS) in nasopharyngeal carcinoma (NPC) was assessed.

Methods: A total of 1,168 non-metastatic NPC patients from Sun Yat-sen University Cancer Center were retrospectively included. The optimal cutoff value for CAR was defined by the Cutoff Finder online tool. Propensity case-matched analysis was performed to adjust for potential differences in baseline characteristics. Subsequently, the prognostic value of the CAR for DMFS was validated in a 756 validation cohort with NPC.

Results: The optimal CAR cutoff value was 0.081 . Patients with high CAR values had significantly poorer DMFS than those with low CAR in univariate and multivariate analyses before propensity score matching. The CAR could also significantly stratify patients into different risks of developing distant metastasis in subgroup analysis. Propensity score analyses showed that CAR remained a prognostic factor for DMFS, thus excluding other interpretations and selection bias. Moreover, the prognostic value of the CAR was robustly confirmed in the external validation cohort.

Conclusion: CAR is an inexpensive and easy-to-measure inflammatory index that may aid clinicians in the development of individualized treatment and follow-up strategies for patients with non-metastatic NPC.

Keywords: nasopharyngeal carcinoma, metastasis, prognosis, propensity score, C-reactive protein, albumin

\section{Introduction}

Nasopharyngeal carcinoma (NPC) is a malignant head and neck cancer with a unique ethnic and geographical distribution; the annual incidence of NPC is as high as 30-80 cases per 10,000 in the southern part of China, Southeast Asia. ${ }^{1}$ The NPC has many sole characteristics to distinguish itself from other head and neck carcinoma. The prominent histological type of NPC is the non-keratinizing carcinoma, which also has association with the Epstein-Barr virus (EBV). Definitive radiotherapy (RT) is recommended as the first-line treatment for non-metastatic NPC due to its higher sensitivity to RT. ${ }^{2}$ The NPC has higher possibility of distant metastasis, and $>20 \%$ of NPC patients ultimately develop distant metastasis even after definitive chemoradiotherapy. ${ }^{3}$ Hence, distant metastasis remains a burning problem to be resolved and a leading cause of death among patients with NPC, especially advanced NPC. ${ }^{4}$ 
The widely used prognostic instrument for stratification and therapeutic decision making in NPC is the tumor node metastasis (TNM) staging classification system. ${ }^{5}$ Various studies tried to discover supplementary molecular biomarkers to the TNM staging to more precisely predict the behavior of NPC to better make a stratification of the NPC prognosis. ${ }^{5,6}$

In recent years, a renaissance of the inflammationcancer connection has occurred, stemming from different lines of work carried out since the 19th century when it was first perceived that cancer is linked to inflammation. ${ }^{7}$ There is increasing evidence to demonstrate that inflammation, both in the tumor microenvironment and systemic circulation, is a key determinant of disease progression and survival in patients with cancer. It has been suggested that cancer-related inflammation is linked to $15-20 \%$ of all cancer-related deaths worldwide, and inflammation has been proposed as the seventh hallmark of cancer., ${ }^{8,9}$ C-Reactive protein (CRP) is a non-specific, acute phase marker of inflammation that has been associated with poorer survival in numerous solid malignancies, such as hepatocellular, colorectal, inoperable non-small-cell lung and breast cancers. ${ }^{10-12}$ CRPs are directly associated with circulating concentrations of vascular endothelial growth factor, contributing to the onset or progression of cancer. ${ }^{13}$ Albumin (ALB) is also an important serum protein that reflects the patients' nutritional status. Hypoalbuminemia, an index of malnutrition and cachexia, is an independent predictor of poor survival in several types of cancers, including NPC. ${ }^{14,15}$

Systemic inflammatory response assessment has been defined by a continuous variable based on CRP and ALB, the CRP/ALB ratio (CAR), which has prognostic value for mortality in patients with sepsis and is associated with survival outcomes in hepatocellular carcinoma, esophageal squamous cell carcinoma and non-small-cell lung cancer. ${ }^{11,12,16}$ Mocellin et al also demonstrated that the CAR could be decreased by dietary supplementation with fish oil in patients with colorectal cancer, which was associated with the prevention of weight loss. However, the prognostic significance of the CAR in NPC has not been studied.

It was hypothesized that immune status, which can be assessed using the CAR, may be associated with distant metastasis-free survival (DMFS) in patients with NPC. Then, the propensity score-matched (PSM) analysis was designed to further validate the CAR prognosis value for DMFS in patients with initially diagnosed NPC.

\section{Patients and methods}

\section{Training cohort}

A total of 1,168 eligible patients with NPC treated at Sun Yat-sen University Cancer Center between October 2007 and December 2009 were retrospectively enrolled. The pre-treatment evaluation included a complete patient history, physical examinations, hematology and biochemistry profiles, magnetic resonance imaging (MRI) of the nasopharynx and neck, chest X-ray, an abdominal sonogram, and a whole-body bone scan. The newly diagnosed, biopsyproven NPC patients without previous anticancer treatment and pathologically proved NPC were included. The patients with distant metastasis or relapsed NPC at initial diagnosis and patients without complete follow-up data were excluded. The study protocol was conducted based on the guidelines outlined in the Declaration of Helsinki, and the study was approved by the ethics committee of Sun Yat-sen University Cancer Center. Written informed consent was obtained from all patients to review their records before the treatment.

All relevant data regarding this study were successfully collected before the treatment, including the personal information and the laboratory (routine blood index), staging (the American Joint Committee on Cancer/International Union for Cancer Control TNM staging system) and therapeutic data. Briefly, serum ALB levels were measured by the bromocresol green (BCG) assay (Sekisui Chemical, Osaka, Japan) according to the manufacturer's instruction. CRP levels were measured by a latex particle-enhanced immunoturbidimetric assay (Sekisui Chemical) according to the manufacturer's instruction.

\section{Treatment}

All patients were treated by definitive RT. The radiation dose ranges to the nasopharynx, lymph node-positive area and lymph node-negative area were 60-80, 60-70 and 50-60 Gy, respectively. In addition, the institutional guidelines recommended no chemotherapy for stage I and II patients and induction, concurrent and adjuvant chemotherapy or combination treatment for stage III and IV patients. Induction or adjuvant chemotherapy incorporates cisplatin with 5-fluorouracil, cisplatin with taxoids or triplet of cisplatin and 5-fluorouracil plus paclitaxel per 3 weeks for 2-3 cycles. Concurrent chemotherapy includes cisplatin given on weeks 1, 4 and 7 of RT or cisplatin given weekly.

\section{Validation cohort}

A validation cohort of 756 consecutive patients with NPC who received definitive RT at the Sun Yat-sen University 
between December 2009 and May 2011 was retrospectively enrolled. Only patients without distant metastasis at initial diagnosis were included; all patients had sufficient data available to assess the prognostic value of the CAR.

\section{Follow-up}

Patients were followed up every 3 months during the first 2 years and every 6 months thereafter until death. The routine assessment for the distant metastasis includes the nasopharyngoscopy, head and neck MRI, abdominal ultrasonography, chest X-rays and/or CT and bone scans per 3 months after the completion of the treatment during the first 3 years and annually thereafter. DMFS was the period from the treatment completion to the time of metastasis or the date of the last follow-up. The last follow-up was on March 30, 2016.

\section{Statistical analysis}

Comparisons between the high CAR and the low CAR were performed using the chi-square test or Fisher's exact test for categorical variables. Continuous variables were converted into dichotomous variables at median value.

An optimal CAR cutoff level was selected using the webbased system Cutoff Finder, which was designed by Budczies et $\mathrm{al}^{17}$ (http://molpath.charite.de/cutoff). The R statistical language acts as an engine for all statistical computing and visualization. ${ }^{18}$ The $\mathrm{R}$ file is loaded as a static source to the $\mathrm{R}$ server. For each cutoff analysis, the web application calls the wrapper $\mathrm{R}$ function get.cutoff() that serves as a controller. The controller calls specialized $\mathrm{R}$ functions for each kind of cutoff optimization and for each kind of plot. Results of the cutoff optimization and plots are returned to the web server. Using the $\mathrm{R}$ code, results and plots of the web page can be reproduced $100 \%$. DMFS curves were created using the Kaplan-Meier method and compared using the log-rank test. The Cox regression analysis was conducted to assess the prognostic significance of CAR to DMFS; significant level of $<0.2$ in the univariate Cox regression analysis was included into the multivariate analysis. To further evaluate the CAR significance to DMFS, the subgroups were applied. The subgroups were divided based on the significant factors in univariate analysis.

To overcome biases introduced by potential varied confounders between the low and high CAR values, PSM was carried out using version 2.9.0R software (R software, Vienna, Austria) via one-to-one matching using MathIt package, which is becoming increasingly used in the medical and surgical literature. ${ }^{19-21}$ The variables incorporated into the propensity matching were the variables correlated with the index of CAR and significant variables in the multivariate
Cox regression of DMFS, in order to not miss the important prognostic factors.

Finally, the prognostic value of CAR was verified in the external validation cohort. The SPSS 19.0 for Windows (IBM Corporation, Armonk, NY, USA) was used for the statistical analyses. Two-sided $P$ values $<0.05$ were considered significant. All data have been deposited at Sun Yat-sen University Cancer Center for future reference (number RDDA2017000365).

\section{Results \\ Patients' characteristics and DMFS}

A total of 1,168 and 756 patients were included in the training and the validation cohort for these analyses. Median DMFS follow-up was 68.8 months in the training data set and 60.25 months in the validation data set. Five-year DMFS in the training data set and validation data set was $85.6 \%$ and $83.5 \%$, respectively. The patient characteristics are summarized in Table 1.

\section{Prognostic value of CAR for DMFS in the I, I 68 patients with NPC and the external cohort who underwent definitive RT}

The median value of CAR in both the training database and external database was 0.032 . The optimal cutoff value was found to be 0.081 using the Cutoff Finder, which produces the greatest distinction of the DMFS in Kaplan-Meier analysis (Figure 1). CAR was prominently associated with gender, smoking status, white blood cell (WBC), neutrophils, alkaline phosphatase (ALP), lactate dehydrogenase (LDH), EBV-DNA, $\mathrm{T}$ and $\mathrm{N}$ classification in the training cohort by the chi-square test (Table 2).

The CAR had the ability to distinguish between patients who developed distant metastasis in the training cohort $(P<0.001)$ and external cohort $(P=0.021$; Figure $2 \mathrm{~A}$ and $\mathrm{B}$, respectively). In the multivariate analysis, the DMFS rates of patients with low and high CAR values remained significantly different $(P<0.001)$. In the training cohort, smoking status $(P=0.031)$, basal serum LDH level $(P=0.026)$, EBV-DNA copy number $(P=0.001), \mathrm{N}$ category $(P<0.001)$, treatment method $(P<0.040)$ and RT technique $(P=0.004)$ were identified as significantly independent prognostic factors related to DMFS (Table 3). In addition, the prognostic value of CAR also exists in different subgroups (Figure 3).

\section{PSM analysis}

PSM yielded a total of 286 matched pairs of patients from the training cohort. The matched covariables include gender, 
Table I Clinical and laboratory characteristics of the patients in the training set and validation set

\begin{tabular}{|c|c|c|}
\hline \multirow[t]{2}{*}{$\overline{\text { Characteristics }}$} & \multirow{2}{*}{$\begin{array}{l}\text { Training set } \\
\text { Number of } \\
\text { cases (\%) }\end{array}$} & \multirow{2}{*}{$\begin{array}{l}\text { Validation test } \\
\text { Number of } \\
\text { cases (\%) }\end{array}$} \\
\hline & & \\
\hline \multicolumn{3}{|l|}{ Age, years } \\
\hline$<45$ & $602(5 I .5)$ & $403(53.3)$ \\
\hline$\geq 45$ & $566(48.5)$ & $352(46.7)$ \\
\hline \multicolumn{3}{|l|}{ Gender } \\
\hline Male & $853(73)$ & $556(73.5)$ \\
\hline Female & $315(27)$ & $200(26.5)$ \\
\hline \multicolumn{3}{|l|}{ Smoking status } \\
\hline Absent & $705(60.4)$ & $498(65.9)$ \\
\hline Present & $463(39.6)$ & $258(34.1)$ \\
\hline \multicolumn{3}{|l|}{ WBC, $\times 10^{9} / \mathrm{L}$} \\
\hline$<6.9$ & $608(52.1)$ & $413(54.6)$ \\
\hline$\geq 6.9$ & $560(47.9)$ & $343(45.4)$ \\
\hline \multicolumn{3}{|l|}{ Neutrophils, $\times 10^{9} / \mathrm{L}$} \\
\hline$<4.1$ & $601(5 I .5)$ & $400(52.9)$ \\
\hline$\geq 4.1$ & $567(48.5)$ & $356(47.1)$ \\
\hline \multicolumn{3}{|l|}{ HGB, g/L } \\
\hline$<143$ & $596(5 I)$ & $369(48.8)$ \\
\hline$\geq 143$ & $572(49)$ & $387(51.2)$ \\
\hline \multicolumn{3}{|l|}{$\mathrm{ALT}, \mathrm{U} / \mathrm{L}$} \\
\hline$<20.6$ & $585(50.1)$ & $358(47.4)$ \\
\hline$\geq 20.6$ & $583(49.9)$ & $398(52.6)$ \\
\hline \multicolumn{3}{|l|}{ AST, U/L } \\
\hline$<20.8$ & $588(50.3)$ & 377 (49.9) \\
\hline$\geq 20.8$ & $580(49.7)$ & $379(50.1)$ \\
\hline \multicolumn{3}{|l|}{ ALP, U/L } \\
\hline$<66.7$ & $591(50.6)$ & $373(49.3)$ \\
\hline$\geq 66.7$ & $577(49.4)$ & $383(50.7)$ \\
\hline \multicolumn{3}{|l|}{$\mathrm{LDH}, \mathrm{U} / \mathrm{L}$} \\
\hline$<166$ & $583(49.9)$ & $379(50.1)$ \\
\hline$\geq 166$ & $585(50.1)$ & 377 (49.9) \\
\hline \multicolumn{3}{|l|}{ EBV-DNA, copies/mL } \\
\hline$<1,000$ & $500(42.8)$ & $382(50.5)$ \\
\hline $1,000-9,999$ & $230(19.7)$ & $144(19.1)$ \\
\hline $10,000-99,999$ & $281(24.1)$ & $152(20.1)$ \\
\hline$>100,000$ & $157(13.4)$ & $78(10.3)$ \\
\hline \multicolumn{3}{|l|}{ CRP, mg/L } \\
\hline$<1.49$ & $583(49.9)$ & $382(50.5)$ \\
\hline$\geq 1.49$ & $585(50.1)$ & $374(49.5)$ \\
\hline \multicolumn{3}{|l|}{$A L B, g / L$} \\
\hline$<45.6$ & $585(50.6)$ & $350(46.3)$ \\
\hline$\geq 45.6$ & $583(49.9)$ & $406(53.7)$ \\
\hline \multicolumn{3}{|l|}{ Radiotherapy technique } \\
\hline CRT & $496(42.5)$ & $498(65.9)$ \\
\hline IMRT + 3DCRT & $672(57.5)$ & $258(34.1)$ \\
\hline \multicolumn{3}{|l|}{ Treatment method } \\
\hline Radiotherapy & $220(18.8)$ & $182(24.1)$ \\
\hline CCRT & $494(42.3)$ & $244(32.2)$ \\
\hline $\mathrm{Neo}+$ radiotherapy & $210(18.0)$ & $152(20.1)$ \\
\hline $\mathrm{Neo}+\mathrm{CCRT}$ & $244(20.9)$ & $178(23.5)$ \\
\hline \multicolumn{3}{|l|}{$\mathrm{T}$ category } \\
\hline I & $76(6.5)$ & $69(9.1)$ \\
\hline 2 & $300(25.7)$ & $17 \mid(22.6)$ \\
\hline 2 & $547(46.8)$ & $358(47.4)$ \\
\hline 3 & $245(21.0)$ & $158(20.9)$ \\
\hline
\end{tabular}

(Continued)
Table I (Continued)

\begin{tabular}{cll}
\hline Characteristics & \begin{tabular}{l} 
Training set \\
\cline { 2 - 3 }
\end{tabular} & $\begin{array}{l}\text { Validation test } \\
\text { cases (\%) }\end{array}$ \\
\hline N category & $\begin{array}{l}\text { Number of } \\
\text { cases (\%) }\end{array}$ \\
\hline 0 & $246(21.1)$ & $162(21.4)$ \\
I & $425(36.4)$ & $305(40.3)$ \\
2 & $310(26.5)$ & $201(26.6)$ \\
3 & $187(16.0)$ & $88(11.6)$ \\
Clinical stage & & \\
I & $29(2.5)$ & $28(3.7)$ \\
II & $199(17)$ & $120(15.9)$ \\
III & $620(53.1)$ & $413(54.6)$ \\
IV & $320(27.4)$ & $195(25.8)$ \\
Distant metastasis & & \\
Absent & $981(84)$ & $627(82.9)$ \\
Present & $187(16.0)$ & $129(17.1)$ \\
Survival status & & \\
Alive & $952(81.5)$ & $632(83.6)$ \\
Dead & $216(18.5)$ & $124(16.4)$ \\
\hline
\end{tabular}

Abbreviations: 3DCRT, three-dimensional conformal radiation therapy; ALB, albumin; ALP, alkaline phosphatase; AST, aspartate transaminase; ALT, alanine transaminase; CAR, CRP/ALB ratio; CCRT, concurrent radiotherapy; CRP, C-reactive protein; $C R T$, conventional radiotherapy; DMFS, distant metastasis-free survival; EBVDNA, Epstein-Barr virus DNA; GLB, globulin; HGB, hemoglobin; IMRT, intensitymodulated radiation therapy; $L D H$, lactate dehydrogenase; Neo, neoadjuvant chemotherapy; RT, radiotherapy; WBC, white blood cell.

smoking status, WBC, ALP, LDH, T staging, N category, EBV-DNA copy number, RT technique and treatment methods. After propensity matching, the distribution of confounding variables was remarkably balanced between the high and low CAR groups, with no significant difference in any clinicopathological factors (Table 2), indicating improved overall balance after matching.

CAR also had the ability to distinguish patients who developed distant metastasis after propensity matching by the log-rank test $(P<0.001$, Figure $2 C)$. Univariate analysis showed that the risk of distant metastasis was greater for the high CAR group than the low CAR group $(P<0.001)$. Gender $(P=0.030)$, LDH level $(P=0.047)$, EBV-DNA copy number $(P=0.007)$ and $\mathrm{N}$ category $(P<0.001)$ were also significantly associated with DMFS in univariate analysis (Table 4).

Multivariate analysis revealed that the CAR remained an independent prognostic factor for DMFS after the propensity score matching within the training cohort $(P=0.001)$. Compared to the low CAR group $(<0.081)$, the high CAR group $(\geq 0.081)$ had a 1.896-times higher risk of distant metastasis (hazard ratio [HR], 1.896; 95\% confidence interval [CI], $1.302-2.763 ; P<0.001)$. N category $(P<0.001)$ and gender $(P=0.014)$ were also independent prognostic factors for DMFS after the propensity score matching (Table 4). 
Significant $(P<0.05)$ tests:1008 out of $1061(95 \% \mathrm{Cl})$

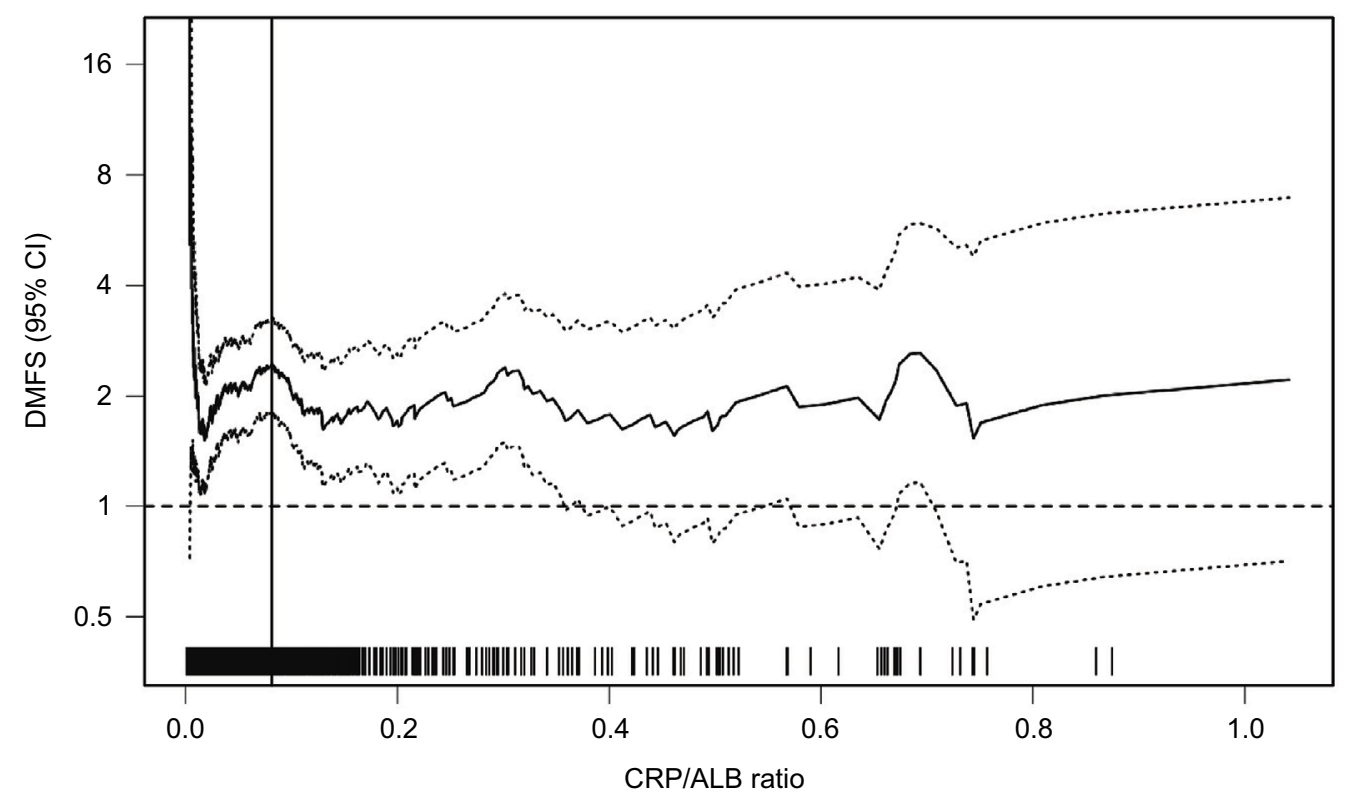

Figure I Hazard ratios (HRs) for distant metastasis-free survival (DMFS) for various cutoff points of the C-reactive protein/albumin (CRP/ALB) ratio (CAR) in patients with nasopharyngeal carcinoma.

Notes: The value of $\mathrm{X}$-axis represented all the possible values of CAR in our patient population. The $\mathrm{Y}$-axis represents $\mathrm{HRs}$. From the left to right of the $\mathrm{X}$-axis, all the possible CAR values were tested as a cutoff for discrimination of DMFS and the corresponding HR and its $95 \%$ confidence interval (Cl) were calculated and plotted (two dashed lines represent upper and lower limits of $95 \% \mathrm{Cls}$, respectively, and the solid line between the two dashed lines represents HR). The vertical line designates the optimal cutoff point with the most significant split (log-rank test). The plots were generated using Cutoff Finder.

\section{Discussion}

As far as we are aware, this is the first assessment of the prognostic value of the CAR with respect to DMFS in patients initially diagnosed with NPC by the PSM method. The CAR was a significant prognostic factor for DMFS, even in subgroup analysis and after PSM analysis, thus the CAR may be a potential novel biomarker for tailored therapy in NPC.

Accumulating evidence indicates that inflammation can facilitate the growth, invasion, metastasis and angiogenesis of cancer cells through inflammatory factors, including acute phase proteins such as CRP, chemokines, cytokines such as interleukin 6 (IL-6), transcription factors such as NF- $\kappa \mathrm{B}$ and circulating and infiltrating immune cells derived from the systemic immune reaction to tumor cells or their secretions. ${ }^{10,22-25}$ Among various markers of inflammation, $\mathrm{CRP}$ is a non-specific acute-phase reactant regulated by proinflammatory cytokines, in particular IL-6 and has also been proved to be associated with inferior survival of numerous malignancies. ${ }^{23,26} \mathrm{An}$ elevated CRP concentration has recently been shown to be inversely associated with T-lymphocyte subset infiltration and an impaired T-lymphocytic response, subverting the host immune response, promoting resistance to cytotoxic drugs and then contributing to a poor survival outcome. ${ }^{27}$ Malnutrition may promote tumor growth and metastasis by damaging the immune system and alter tumor cell biology in the tumor microenvironment. ${ }^{15,28}$ ALB is an important serum protein that reflects the patients' nutritional status and was recently recommended as an objective prognostic factor for poorer survival in various types of cancers including NPC, and ALB is the most clinically used index to value the nutritional status of the patients. ${ }^{29}$ It has been been speculated that higher ALB level could strengthen the human defense mechanism including the cellar immunity, humoral immunity, and cell phagocytic function and be the mechanism of malnutrition prognosis..$^{30-32}$ The enhanced immunology could also boost susceptibility to infection and further reduce the adverse reaction to treatment. ${ }^{30}$ There are also studies that suggest that the higher ALB could weaken the cell growth rate and DNA replication and stabilize the routine biochemical index changes, such as calcium level. ${ }^{41,42}$ Moreover, the stable sex hormone level contributes to defense against sex hormone-related cancers. The antioxidant effect is another attribute against carcinogens, including nitrosamine and aflatoxin. ${ }^{30,32}$

Moreover, the presence of a systemic inflammatory response, as indicated by an elevated CRP level and hypoalbuminemia, accompanies progressive loss of weight and lean tissue, resulting in poor performance status and higher rate of 
Table 2 Associations between CAR and clinicopathological features before and after propensity score matching

\begin{tabular}{|c|c|c|c|c|c|c|}
\hline \multirow[t]{2}{*}{ Characteristics } & \multicolumn{3}{|c|}{ CAR (before matching) } & \multicolumn{3}{|c|}{ CAR (after matching) } \\
\hline & Low, n (\%) & High, n (\%) & $P$ & Low, n (\%) & High, n (\%) & $P$ \\
\hline \multicolumn{7}{|l|}{ Age, years } \\
\hline$<45$ & $419(75.9)$ & $133(24.1)$ & $<0.768$ & III (45.5) & $133(54.5)$ & 0.063 \\
\hline$\geq 45$ & $463(75.2)$ & $153(24.8)$ & & $175(53.4)$ & $153(46.6)$ & \\
\hline \multicolumn{7}{|l|}{ Gender } \\
\hline Male & 630 (73.9) & $223(26.1)$ & 0.030 & $225(50.2)$ & $223(49.8)$ & 0.839 \\
\hline Female & $252(80.0)$ & $63(20.2)$ & & $61(49.2)$ & $63(50.8)$ & \\
\hline \multicolumn{7}{|l|}{ Smoking status } \\
\hline No & $545(77.3)$ & $160(22.7)$ & 0.079 & $152(48.7)$ & $160(51.3)$ & 0.502 \\
\hline Yes & $337(72.8)$ & $126(27.2)$ & & $134(5 \mid .5)$ & $126(48.4)$ & \\
\hline \multicolumn{7}{|l|}{ WBC, $\times 10^{9}$} \\
\hline$<6.9$ & $504(82.9)$ & $104(17.1)$ & $<0.001$ & $98(48.5)$ & $104(51.5)$ & 0.600 \\
\hline$\geq 6.9$ & $378(67.5)$ & $182(32.5)$ & & $188(50.8)$ & $182(49.2)$ & \\
\hline \multicolumn{7}{|l|}{ Neutrophils, $\times 10^{9}$} \\
\hline$<4.1$ & $368(6 I .3)$ & $233(38.7)$ & 0.026 & $107(50)$ & $107(50)$ & 1.000 \\
\hline$\geq 4.1$ & $28 I(50.5)$ & $286(49.5)$ & & $179(50.0)$ & $179(50.0)$ & \\
\hline \multicolumn{7}{|l|}{$\mathrm{HGB}, \mathrm{g} / \mathrm{L}$} \\
\hline$<143$ & $440(73.8)$ & $156(26.2)$ & 0.171 & $158(50.3)$ & $156(49.7)$ & 0.867 \\
\hline$\geq 143$ & $442(77.3)$ & $130(22.7)$ & & $128(49.6)$ & $130(50.4)$ & \\
\hline \multicolumn{7}{|l|}{$\mathrm{ALT}, \mathrm{U} / \mathrm{L}$} \\
\hline$<20.6$ & $445(76.1)$ & 140 (23.9) & 0.659 & $154(52.4)$ & $140(47.6)$ & 0.242 \\
\hline$\geq 20.6$ & $437(75.0)$ & $146(25.0)$ & & $132(47.5)$ & $146(52.5)$ & \\
\hline \multicolumn{7}{|l|}{ AST, U/L } \\
\hline$<20.8$ & $446(75.9)$ & $142(24.1)$ & 0.788 & $146(50.7)$ & $142(49.3)$ & 0.738 \\
\hline$\geq 20.8$ & $436(75.2)$ & $144(24.8)$ & & $140(49.3)$ & $144(50.7)$ & \\
\hline \multicolumn{7}{|l|}{ ALP, U/L } \\
\hline$<66.7$ & $490(82.9)$ & $101(17.1)$ & $<0.001$ & $107(5 \mathrm{I} .4)$ & $101(48.6)$ & 0.602 \\
\hline$\geq 66.7$ & $392(67.9)$ & $185(32.1)$ & & $179(49.2)$ & $185(50.8)$ & \\
\hline \multicolumn{7}{|l|}{$\mathrm{LDH}, \mathrm{U} / \mathrm{L}$} \\
\hline$<166$ & $470(80.6)$ & $113(19.4)$ & $<0.001$ & $116(50.7)$ & $113(49.3)$ & 0.798 \\
\hline$\geq 166$ & $412(70.4)$ & $173(29.6)$ & & $170(49.6)$ & $173(50.4)$ & \\
\hline \multicolumn{7}{|l|}{ EBV-DNA, copies/mL } \\
\hline$<1,000$ & $394(78.8)$ & $106(21.2)$ & 0.002 & $110(50.9)$ & $106(49.1)$ & 0.146 \\
\hline $1,000-9,999$ & $183(79.6)$ & $47(20.4)$ & & $60(56.1)$ & $47(43.9)$ & \\
\hline $10,000-99,999$ & $190(67.6)$ & 91 (32.4) & & $68(42.8)$ & 91 (57.2) & \\
\hline$>100,000$ & 115 (73.2) & $42(26.8)$ & & $48(53.3)$ & $42(46.7)$ & \\
\hline \multicolumn{7}{|l|}{ Radiotherapy technique } \\
\hline CRT & $507(75.4)$ & $165(24.6)$ & 0.950 & $122(50.2)$ & 121 (49.8) & 0.933 \\
\hline IMRT + 3DCRT & $375(75.6)$ & $|2|(24.4)$ & & $164(49.8)$ & $165(50.2)$ & \\
\hline \multicolumn{7}{|l|}{ Treatment method } \\
\hline Radiotherapy & I7I (77.7) & $49(22.3)$ & 0.103 & $36(42.2)$ & $49(57.6)$ & 0.113 \\
\hline CCRT & $383(77.5)$ & III (22.5) & & $137(55.2)$ & III (44.8) & \\
\hline $\mathrm{Neo}+$ radiotherapy & $158(75.2)$ & $52(24.8)$ & & $52(50.0)$ & $52(50.0)$ & \\
\hline $\mathrm{Neo}+\mathrm{CCRT}$ & $170(69.7)$ & $74(30.3)$ & & $61(45.2)$ & $74(54.8)$ & \\
\hline \multicolumn{7}{|l|}{$\mathrm{T}$ category } \\
\hline I & $62(81.6)$ & $14(18.4)$ & $<0.001$ & $10(4 \mid .7)$ & $14(58.3)$ & 0.765 \\
\hline 2 & $249(83.0)$ & $51(17.0)$ & & $53(5 \mathrm{I} .0)$ & $51(49.0)$ & \\
\hline 3 & $416(76.1)$ & $|3|$ (23.9) & & $139(5 \mid .5)$ & $131(48.5)$ & \\
\hline 4 & $155(63.3)$ & $90(36.7)$ & & $84(48.3)$ & $90(5 \mid .7)$ & \\
\hline \multicolumn{7}{|l|}{$N$ category } \\
\hline 0 & $210(81.7)$ & $45(18.3)$ & 0.005 & $4 \mathrm{I}(47.7)$ & $45(52.3)$ & 0.578 \\
\hline 1 & $321(75.5)$ & $104(24.5)$ & & $106(50.5)$ & $104(49.5)$ & \\
\hline 2 & $235(75.8)$ & $75(24.2)$ & & $87(53.7)$ & $75(46.3)$ & \\
\hline 3 & $125(66.8)$ & $62(33.2)$ & & $52(45.6)$ & $62(54.4)$ & \\
\hline
\end{tabular}

Abbreviations: 3DCRT, three-dimensional conformal radiation therapy; ALB, albumin; ALP, alkaline phosphatase; AST, aspartate transaminase; ALT, alanine transaminase; CAR, CRPIALB ratio; CCRT, concurrent radiotherapy; CRP, C-reactive protein; CRT, conventional radiotherapy; DMFS, distant metastasis-free survival; EBV-DNA, Epstein-Barr virus DNA; GLB, globulin; HGB, hemoglobin; IMRT, intensity-modulated radiation therapy; LDH, lactate dehydrogenase; Neo, neoadjuvant chemotherapy; RT, radiotherapy; WBC, white blood cell. 

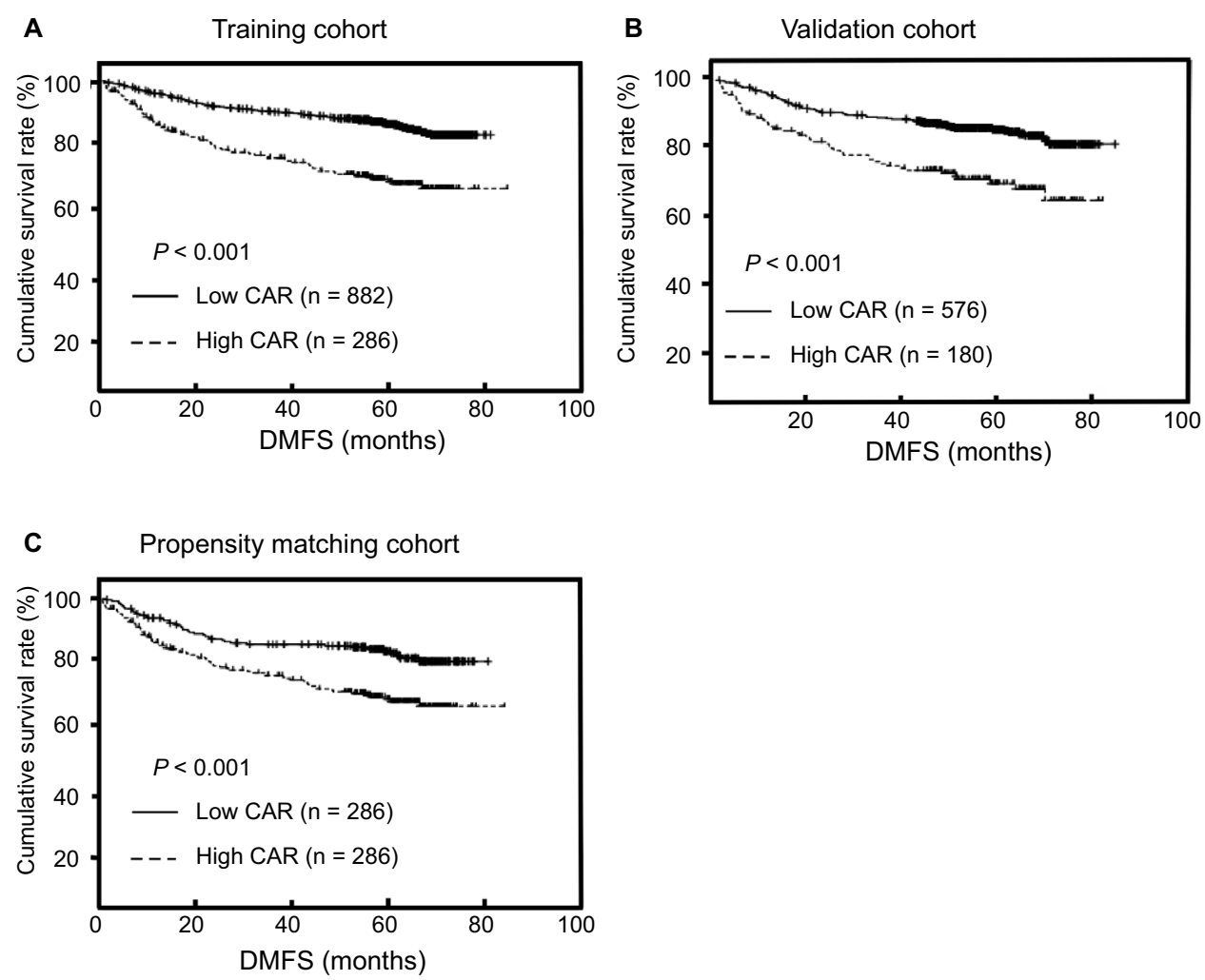

Figure 2 Prognostic value of the C-reactive protein/albumin ratio (CAR) for distant metastasis-free survival (DMFS) in the training cohort before matching (A), the validation cohort (B) and training cohort after I:I ratio matching (C).

mortality in patients with cancer. ${ }^{12,33}$ As a single factor alone may not accurately predict prognosis, inflammatory indexes that combine CRP and serum ALB, such as the CAR, may have more significant prognostic value. Ranzani et $\mathrm{al}^{34}$ first proposed the concept of CAR and demonstrated its value for mortality of septic patients. Moreover, CRP and ALB are sensitive and reliable immunonutritional markers that can be conveniently assessed in clinical laboratories using standardized parameters.

Glasgow prognostic score or modified Glasgow prognostic score indexes which combine serum CRP and ALB levels have also been viewed as prognostic factors of NPC. ${ }^{32}$ However, the modified Glasgow prognostic score is a qualitative index, and the majority of initially diagnosed NPC patients have good performance and nutritional status. Therefore, the diagnosis of hypoalbuminemia before treatment restricts the application of this index. By contrast, the CAR is a continuous variable, which could reduce the potential for systemic inflammation and nutritional status overestimation or underestimation and more precisely stratify patient's survival.

In this study, univariate analysis showed that an elevated CAR $(\geq 0.081)$ was associated with poorer DMFS in stages I-III NPC. Additionally, the binary valuable CAR was prominently related to other inflammatory factors such as
WBC, ALP and LDH, indicating that the CAR may also carry their prognostic value, thus bringing in the potential prognostic interference. Then, PSM analysis is used, a tool used to adjust a group effect for measured confounders in non-randomized studies and to avoid confounding bias. The CAR was independently associated with DMFS in multivariate analysis. Moreover, subgroup analysis confirmed the CAR prognostic guidance value for DMFS in non-metastasis NPC. Therefore, these results demonstrate that high CAR, a chronic systemic index reflecting malnutrition and inflammatory state, is a poorer survival index in NPC. Therefore, our results indicate that the CAR may emphasize the CAR significance for DMFS in NPC.

Intriguingly, non-steroidal anti-inflammatory agents have been reported to reduce mortality in several tumor types. ${ }^{35}$ Regulation of the immune system is a novel target that has been proposed as a method of improving the treatment of cancer, and the CAR may represent a useful indicator of the immune response and enhance the effectiveness of such strategies. It is possible that nutritional therapy, such as the administration of branched-chain amino acid-enriched nutrient support, may improve the CAR and therefore potentially affect the rate of DMFS in patients with NPC. ${ }^{36}$ 
Table 3 Univariate and multivariate analyses of associations between CAR and DMFS before propensity score matching in the training cohort

\begin{tabular}{|c|c|c|c|c|c|c|}
\hline \multirow[t]{2}{*}{ Characteristics } & \multicolumn{3}{|c|}{ Univariate } & \multicolumn{3}{|c|}{ Multivariate } \\
\hline & HR & $95 \% \mathrm{Cl}$ & $P$ & HR & $95 \% \mathrm{Cl}$ & $P$ \\
\hline Age ( $\geq 45$ years vs $<45$ ) & 1.138 & $0.853-1.519$ & 0.379 & & & \\
\hline Gender (female vs male) & 0.673 & $0.473-0.958$ & 0.028 & & & \\
\hline Smoking status (present vs absent) & 1.484 & $1.113-1.979$ & 0.007 & 1.376 & $1.030-1.839$ & 0.031 \\
\hline WBC, $\times 10^{9}(\geq 6.9$ vs $<6.9)$ & 1.180 & $0.885-1.573$ & 0.260 & & & \\
\hline Neutrophils, $\times 10^{9}(\geq 4.1$ vs $<4.1)$ & 1.213 & $0.782-1.883$ & 0.388 & & & \\
\hline NLR, (>I.05 vs <l.05) & 1.799 & $1.238-2.615$ & 0.002 & 1.197 & $0.811-1.767$ & 0.366 \\
\hline HGB, g/L ( $\geq 143$ vs $<143)$ & 1.076 & $0.807-1.434$ & 0.617 & & & \\
\hline ALT, U/L ( $\geq 20.6$ vs $<20.6)$ & 1.260 & $0.944-1.683$ & 0.117 & & & \\
\hline AST, U/L ( $\geq 20.8$ vs $<20.8)$ & 1.237 & $0.927-1.65 \mid$ & 0.148 & & & \\
\hline ALP, U/L ( $\geq 66.7$ vs <66.7) & 1.148 & $0.86 I-1.530$ & 0.348 & & & \\
\hline $\mathrm{LDH}, \mathrm{U} / \mathrm{L}(\geq 166$ vs $<166)$ & 1.712 & $1.275-2.300$ & $<0.001$ & 1.408 & $1.043-1.902$ & 0.026 \\
\hline EBV-DNA, copies/mL & & & $<0.001$ & & & 0.001 \\
\hline$<1,000$ & 1.000 & & & 1.000 & & \\
\hline $1,000-9,999$ & 1.190 & $0.764-1.854$ & 0.443 & 1.079 & $0.690-1.687$ & 0.740 \\
\hline $10,000-99,999$ & 1.799 & $1.238-2.615$ & 0.002 & 1.350 & $0.917-1.986$ & 0.128 \\
\hline$>100,000$ & 2.843 & $1.924-4.200$ & $<0.001$ & 2.276 & $1.515-3.420$ & $<0.001$ \\
\hline T category & & & 0.003 & & & \\
\hline 1 & 1.000 & & & & & \\
\hline 2 & 0.745 & $0.378-|.47|$ & 0.397 & & & \\
\hline 3 & 1.053 & $0.562-1.973$ & 0.872 & & & \\
\hline 4 & 1.635 & $0.856-3.125$ & 0.137 & & & \\
\hline $\mathrm{N}$ category & & & $<0.001$ & & & $<0.001$ \\
\hline 0 & 1.000 & & & 1.000 & & \\
\hline 1 & 1.750 & $1.024-2.992$ & 0.041 & 1.562 & $0.906-2.693$ & 0.109 \\
\hline 2 & 2.681 & $1.574-4.565$ & $<0.001$ & 2.144 & $1.231-3.736$ & 0.007 \\
\hline 3 & 6.203 & $3.658-10.518$ & $<0.001$ & 4.730 & $2.698-8.293$ & $<0.001$ \\
\hline Treatment method & & & 0.020 & & & 0.040 \\
\hline Radiotherapy & 1.000 & & & 1.000 & & \\
\hline CCRT & 0.554 & $0.327-0.937$ & 0.028 & 1.064 & $0.639-1.773$ & 0.811 \\
\hline $\mathrm{Neo}+$ radiotherapy & 1.009 & $0.693-1.470$ & 0.961 & 1.450 & $0.834-2.521$ & 0.188 \\
\hline $\mathrm{Neo}+\mathrm{CCRT}$ & 1.272 & $0.829-1.95 \mid$ & $0.27 I$ & 0.752 & $0.426-1.325$ & 0.323 \\
\hline Radiotherapy technique (IMRT+3DCRT vs CRT) & 0.715 & $0.536-0.953$ & 0.022 & 0.629 & $0.458-0.865$ & 0.004 \\
\hline CAR $(\geq 0.08 \mathrm{I}$ vs $<0.08 \mathrm{I})$ & $2.46 I$ & $1.835-3.300$ & $<0.001$ & 2.063 & $1.526-2.788$ & $<0.001$ \\
\hline
\end{tabular}

Abbreviations: 3DCRT, three-dimensional conformal radiation therapy; ALB, albumin; ALP, alkaline phosphatase; AST, aspartate transaminase; ALT, alanine transaminase; CAR, CRPIALB; CCRT, concurrent radiotherapy; CRP, C-reactive protein; Cl, confidence interval; CRT, conventional radiotherapy; DMFS, distant metastasis-free survival; EBV-DNA, Epstein-Barr virus DNA; GLB, globulin; HGB, hemoglobin; HR, hazard ratio; IMRT, intensity-modulated radiation therapy; LDH, lactate dehydrogenase; Neo, neoadjuvant chemotherapy; NLR, neutrophil to lymphocyte ratio; RT, radiotherapy; WBC, white blood cell.

The suitable cutoff value of the CRP/ALB is different in different studies, even in the same tumor type. In the two studies of NPC, the cutoff values were different. In the study by Sun et al,,$^{37}$ the cutoff was set to be 0.189 , which was slightly higher than that in the study by Tao et $\mathrm{al}^{38}(0.141)$, owing to the different methods and/or different patients enrolled in the study. In our study, a more novel and reliable method was used and a cutoff value was determined to be 0.089 , which is similar to that of the study by Zhang et al. ${ }^{39}$ The prognostic significance was also validated in an external cohort of 756 patients.

The limitations of this study should be acknowledged. This was a retrospective study and a selection bias may exist. However, we enrolled a relatively large training cohort (1,168 patients) to evaluate the value of CAR for DMFS. Additionally, the PSM was also conducted to reduce the potential confounders, and the prognostic value of the CAR was also validated in the validation cohort (756 patients). However, additional assessment of patients from other centers and prospective data sets is necessary. CRP is a sensitive cancer marker but lacks specificity and other non-cancerous influences were not excluded (infections, surgery, and connective tissue disease). However, the limitation were overcome, since the routine blood index was collected before treatment. 


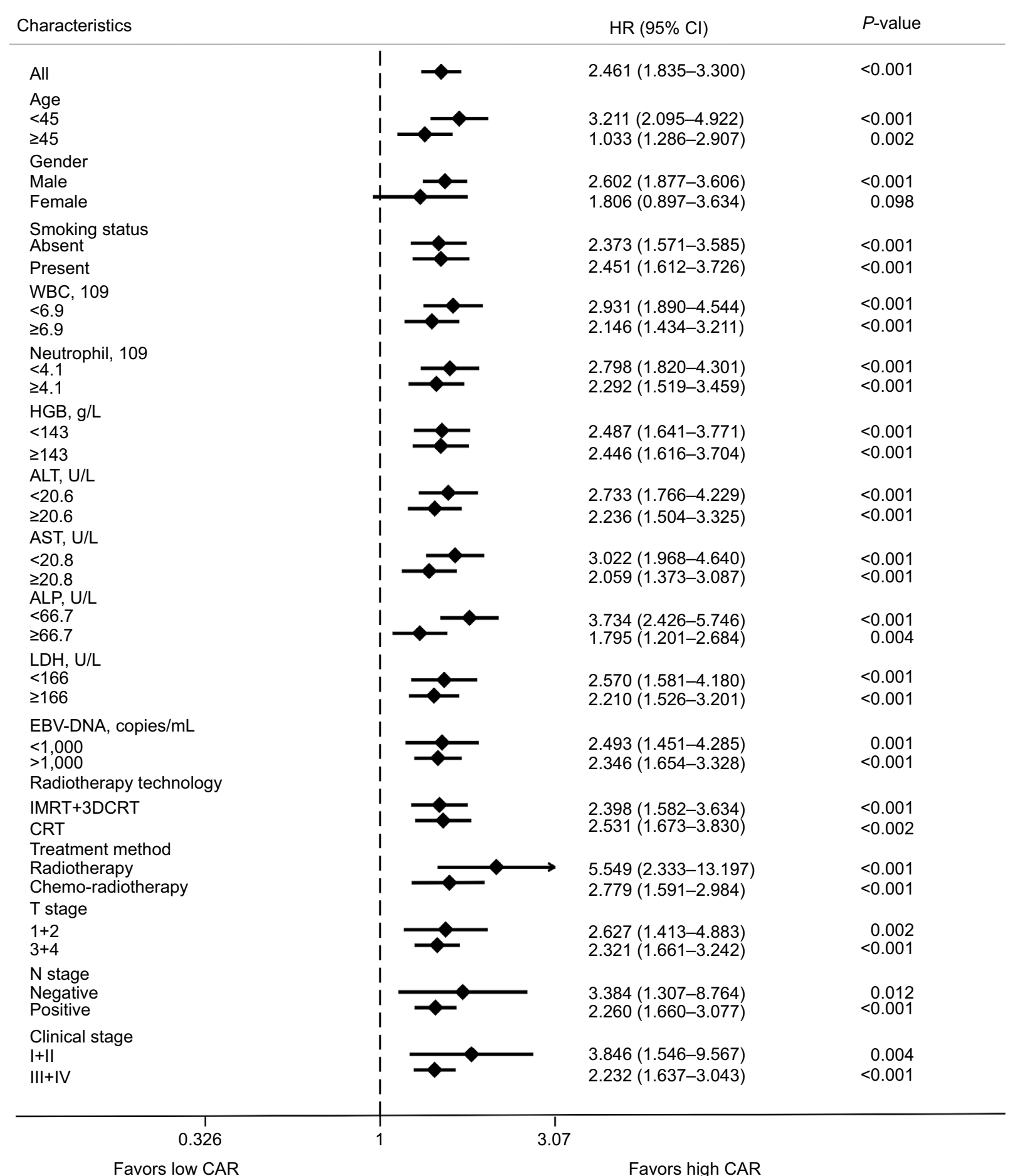

Figure 3 Forest plot of subgroup analysis showing hazard ratios (HRs) and $95 \% \mathrm{Cl}$ (bars) for DMFS in I, 168 patients with nasopharyngeal carcinoma who underwent definitive radiotherapy.

Note: Subgroups were defined by factors showing significant associations with the C-reactive protein/albumin ratio (CAR) and DMFS.

Abbreviations: 3DCRT, three-dimensional conformal radiation therapy; ALB, albumin; ALP, alkaline phosphatase; ALT, alanine transaminase; AST, aspartate transaminase; CAR, CRP/ALB ratio; Cl, confidence interval; CRP, C-reactive protein; CRT, conventional radiotherapy; chemo-radiotherapy, chemotherapy plus radiotherapy; DMFS, distant metastasis-free survival; EBV-DNA, Epstein-Barr virus DNA; GLB, globulin; HGB, hemoglobin; IMRT, intensity-modulated radiation therapy; LDH, lactate dehydrogenase; RT, radiotherapy; WBC, white blood cell.

\section{Conclusion}

This study indicates that the CAR represents a potential prognostic factor to more accurately predict patient outcomes in NPC. Quantification of the CAR before treatment could complement current staging methods to improve disease staging and treatment allocation. 
Table 4 Univariate and multivariate analyses of associations between CAR and DMFS after I:I ratio propensity score matching in the training cohort

\begin{tabular}{|c|c|c|c|c|c|c|}
\hline \multirow[t]{2}{*}{ Characteristics } & \multicolumn{3}{|c|}{ Univariate } & \multicolumn{3}{|c|}{ Multivariate } \\
\hline & HR & $95 \% \mathrm{Cl}$ & $P$ & HR & $95 \% \mathrm{Cl}$ & $P$ \\
\hline Age ( $\geq 45$ years vs $<45$ ) & 0.813 & $0.566-1.167$ & $0.26 \mathrm{I}$ & & & \\
\hline Gender (female vs male) & 0.566 & $0.339-0.946$ & 0.030 & 0.525 & $0.314-0.879$ & 0.014 \\
\hline Smoking status (present vs absent) & 1.289 & $0.898-1.849$ & 0.168 & & & \\
\hline WBC, $\times 10^{9}(\geq 6.9$ vs $<6.9)$ & 0.925 & $0.637-1.344$ & 0.683 & & & \\
\hline HGB, g/L ( $\geq 143$ vs $<143)$ & 1.096 & $0.764-1.574$ & 0.618 & & & \\
\hline ALT, U/L $(\geq 20.6$ vs $<20.6)$ & 1.125 & $0.784-1.614$ & 0.524 & & & \\
\hline AST, U/L ( $\geq 20.8$ vs $<20.8)$ & 1.161 & $0.809-1.667$ & 0.417 & & & \\
\hline ALP, U/L ( $\geq 66.7$ vs $<66.7$ ) & 0.800 & $0.554-1.154$ & 0.233 & & & \\
\hline $\mathrm{LDH}, \mathrm{U} / \mathrm{L}(\geq 166$ vs $<166)$ & $\mathrm{I} .474$ & $1.005-2.164$ & 0.047 & & & \\
\hline EBV-DNA, copies/mL & & & 0.007 & & & \\
\hline$<1,000$ & 1.000 & & & & & \\
\hline $1,000-9,999$ & 1.154 & $0.656-2.029$ & 0.620 & & & \\
\hline $10,000-99,999$ & 1.613 & $1.009-2.579$ & 0.046 & & & \\
\hline$>100,000$ & 2.314 & $1.404-3.812$ & 0.001 & & & \\
\hline $\mathrm{T}$ category & & & 0.786 & & & \\
\hline I & 1.000 & & & & & \\
\hline 2 & 0.846 & $0.316-2.266$ & 0.740 & & & \\
\hline 3 & 0.942 & $0.377-2.354$ & 0.899 & & & \\
\hline 4 & 1.106 & $0.436-2.807$ & 0.832 & & & \\
\hline $\mathrm{N}$ category & & & $<0.001$ & & & $<0.001$ \\
\hline 0 & 1.000 & & & 1.000 & & \\
\hline I & 1.039 & $0.517-2.088$ & 0.914 & 1.140 & $0.567-2.293$ & 0.713 \\
\hline 2 & 1.660 & $0.837-3.294$ & 0.147 & 1.888 & $0.949-3.755$ & 0.079 \\
\hline 3 & 4.402 & $2.280-8.499$ & $<0.001$ & 4.675 & $2.419-9.036$ & $<0.001$ \\
\hline Treatment method & & & 0.135 & & & \\
\hline Radiotherapy & 1.000 & & & & & \\
\hline CCRT & 1.392 & $0.756-2.563$ & 0.288 & & & \\
\hline $\mathrm{Neo}+$ radiotherapy & 2.041 & $1.061-3.928$ & 0.033 & & & \\
\hline $\mathrm{Neo}+\mathrm{CCRT}$ & 1.304 & $0.670-2.537$ & 0.435 & & & \\
\hline Radiotherapy technique (IMRT+3DCRT vs CRT) & 0.770 & $0.537-1.105$ & 0.156 & & & \\
\hline $\operatorname{CAR}(\geq 0.08 \mathrm{I}$ vs $<0.08 \mathrm{I})$ & 1.940 & $1.333-2.822$ & 0.001 & 1.896 & $1.302-2.763$ & 0.001 \\
\hline
\end{tabular}

Abbreviations: 3DCRT, three-dimensional conformal radiation therapy; ALB, albumin; ALP, alkaline phosphatase; ALT, alanine transaminase; AST, aspartate transaminase; CAR, CRPIALB; Cl, confidence interval; CCRT, concurrent radiotherapy; CRP, C-reactive protein; CRT, conventional radiotherapy; DMFS, distant metastasis-free survival; EBV-DNA, Epstein-Barr virus DNA; GLB, globulin; HGB, hemoglobin; HR, hazard ratio; IMRT, intensity-modulated radiation therapy; LDH, lactate dehydrogenase; Neo, neoadjuvant chemotherapy; Multi., multivariate; NLR, neutrophil to lymphocyte ratio; RT, radiotherapy; Uni., univariate; WBC, white blood cell.

\section{Availability of data and material}

Raw data was deposited in the Research Data Deposit system, RDDA2017000365 (http://www.researchdata.org.cn) of Sun Yat-sen University Cancer and can be obtained from the corresponding authors on reasonable request.

\section{Author contributions}

WY, XLP, and CY designed the overall project. WY analyzed the data and wrote the manuscript. YL collected and analyzed the data. XLP and CY performed the research. All authors contributed toward data analysis, drafting and critically revising the paper, and agree to be accountable for all aspects of the work.

\section{Disclosure}

The authors report no conflicts of interest in this work.

\section{References}

1. Thompson MP, Kurzrock R. Epstein-Barr virus and cancer. Clin Cancer Res. 2004;10(3):803-821.

2. Lee AW, Ng WT, Chan YH, Sze H, Chan C, Lam TH. The battle against nasopharyngeal cancer. Radiother Oncol. 2012;104(3):272-278.

3. Bensouda Y, Kaikani W, Ahbeddou N, et al. Treatment for metastatic nasopharyngeal carcinoma. Eur Ann Otorhinolaryngol Head Neck Dis. 2011;128(2):79-85.

4. Lai SZ, Li WF, Chen L, et al. How does intensity-modulated radiotherapy versus conventional two-dimensional radiotherapy influence the treatment results in nasopharyngeal carcinoma patients? Int J Radiat Oncol Biol Phys. 2011;80(3):661-668.

5. Wei WI, Sham JS. Nasopharyngeal carcinoma. Lancet. 2005; 365(9476):2041-2054.

6. Ludwig JA, Weinstein JN. Biomarkers in cancer staging, prognosis and treatment selection. Nat Rev Cancer. 2005;5(11):845-856.

7. Coussens LM, Werb Z. Inflammation and cancer. Nature. 2002; 420(6917):860-867.

8. Colotta F, Allavena P, Sica A, Garlanda C, Mantovani A. Cancer-related inflammation, the seventh hallmark of cancer: links to genetic instability. Carcinogenesis. 2009;30(7):1073-1081. 
9. Hanahan D, Weinberg RA. Hallmarks of cancer: the next generation. Cell. 2011;144(5):646-674.

10. Allin KH, Nordestgaard BG. Elevated C-reactive protein in the diagnosis, prognosis, and cause of cancer. Crit Rev Clin Lab Sci. 2011; 48(4):155-170.

11. Kinoshita A, Onoda H, Imai N, et al. The C-reactive protein/albumin ratio, a novel inflammation-based prognostic score, predicts outcomes in patients with hepatocellular carcinoma. Ann Surg Oncol. 2015;22(3):803-810.

12. McMillan DC. The systemic inflammation-based Glasgow Prognostic Score: a decade of experience in patients with cancer. Cancer Treat Rev. 2013;39(5):534-540.

13. Xavier P, Belo L, Beires J, et al. Serum levels of VEGF and TNF-alpha and their association with $\mathrm{C}$-reactive protein in patients with endometriosis. Arch Gynecol Obstet. 2006;273(4):227-231.

14. Du XJ, Tang LL, Mao YP, et al. The pretreatment albumin to globulin ratio has predictive value for long-term mortality in nasopharyngeal carcinoma. PLoS One. 2014;9(4):e94473.

15. McMillan DC. Systemic inflammation, nutritional status and survival in patients with cancer. Curr Opin Clin Nutr Metab Care. 2009;12(3):223-226.

16. Wei XL, Wang FH, Zhang DS, et al. A novel inflammation-based prognostic score in esophageal squamous cell carcinoma: the $\mathrm{C}$-reactive protein/albumin ratio. BMC Cancer. 2015;15(1):350.

17. Budczies J, Klauschen F, Sinn BV, et al. Cutoff Finder: a comprehensive and straightforward Web application enabling rapid biomarker cutoff optimization. PLoS One. 2012;7(12):e51862.

18. Dean CB, Nielsen JD. Generalized linear mixed models: a review and some extensions. Lifetime Data Anal. 2007;13(4):497-512.

19. Hemmila MR, Birkmeyer NJ, Arbabi S, Osborne NH, Wahl WL, Dimick JB. Introduction to propensity scores: a case study on the comparative effectiveness of laparoscopic vs open appendectomy. Arch Surg. 2010;145(10):939-945.

20. Stukel TA, Fisher ES, Wennberg DE, Alter DA, Gottlieb DJ, Vermeulen MJ. Analysis of observational studies in the presence of treatment selection bias: effects of invasive cardiac management on AMI survival using propensity score and instrumental variable methods. JAMA. 2007;297(3):278-285.

21. Tewari A, Divine G, Chang P, et al. Long-term survival in men with high grade prostate cancer: a comparison between conservative treatment, radiation therapy and radical prostatectomy - a propensity scoring approach. J Urol. 2007;177(3):911-915.

22. Balkwill FR. The chemokine system and cancer. J Pathol. 2012; 226(2):148-157.

23. Guthrie GJ, Roxburgh CS, Horgan PG, McMillan DC. Does interleukin-6 link explain the link between tumour necrosis, local and systemic inflammatory responses and outcome in patients with colorectal cancer? Cancer Treat Rev. 2013;39(1):89-96.

24. Hoesel B, Schmid JA. The complexity of NF-kappaB signaling in inflammation and cancer. Mol Cancer. 2013;12:86.

25. Elinav E, Nowarski R, Thaiss CA, Hu B, Jin C, Flavell RA. Inflammation-induced cancer: crosstalk between tumours, immune cells and microorganisms. Nat Rev Cancer. 2013;13(11):759-771.
26. Pepys MB, Hirschfield GM. C-reactive protein: a critical update. J Clin Invest. 2003;111(12):1805-1812.

27. Canna K, McArdle PA, McMillan DC, et al. The relationship between tumour T-lymphocyte infiltration, the systemic inflammatory response and survival in patients undergoing curative resection for colorectal cancer. Br J Cancer. 2005;92(4):651-654.

28. Aoyagi T, Terracina KP, Raza A, Matsubara H, Takabe K. Cancer cachexia, mechanism and treatment. World J Gastrointestinal Oncol. 2015;7(4):17-29.

29. Hu JY, Yi W, Xia YF, Gao J, Liu ZG, Tao YL. [Impact of pretherapy body mass index on prognosis of nasopharyngeal carcinoma]. Ai Zheng. 2009;28(10):1043-1048.

30. Chandra RK. Nutrition and immunology: from the clinic to cellular biology and back again. Proc Nutr Soc. 1999;58(3):681-683.

31. Laursen I, Briand P, Lykkesfeldt AE. Serum albumin as a modulator on growth of the human breast cancer cell line, MCF-7. Anticancer Res. 1990;10(2a):343-351.

32. Sonnenschein C, Soto AM, Michaelson CL. Human serum albumin shares the properties of estrocolyone-I, the inhibitor of the proliferation of estrogen-target cells. J Steroid Biochem Mol Biol. 1996;59(2):147-154.

33. Roxburgh CS, McMillan DC. Cancer and systemic inflammation: treat the tumour and treat the host. Br J Cancer. 2014;110(6):1409-1412.

34. Ranzani OT, Zampieri FG, Forte DN, Azevedo LC, Park M. C-reactive protein/albumin ratio predicts 90 -day mortality of septic patients. PLoS One. 2013;8(3):e59321.

35. Chan AT, Ogino S, Fuchs CS. Aspirin and the risk of colorectal cancer in relation to the expression of COX-2. N Engl J Med. 2007; 356(21):2131-2142.

36. Okabayashi T, Nishimori I, Sugimoto T, et al. Effects of branched-chain amino acids-enriched nutrient support for patients undergoing liver resection for hepatocellular carcinoma.J Gastroenterol Hepatol. 2008;23(12):1869-1873.

37. Sun $\mathrm{P}, \mathrm{Chen} \mathrm{C}, \mathrm{Xia} \mathrm{Y}$, et al. The ratio of $\mathrm{C}$-reactive protein/albumin is a novel inflammatory predictor of overall survival in cisplatin-based treated patients with metastatic nasopharyngeal carcinoma. Dis Markers. 2017;2017:6570808.

38. Tao CJ, Chen YY, Jiang F, et al. The C-reactive Protein/Albumin Ratio Is an independent Prognostic Factor for Overall Survival in Patients with Nasopharyngeal Carcinoma Receiving Intensity-Modulated Radiotherapy. J Cancer. 2016;7(14):2005-2011.

39. Zhang Y, Zhou GQ, Liu X, et al. Exploration and validation of C-reactive protein/albumin ratio as a novel inflammation-based prognostic marker in nasopharyngeal carcinoma. J Cancer. 2016;7(11):1406-1412.

40. A meta-analysis of $n-3$ polyunsaturated fatty acids effects on circulating acute-phase protein and cytokines in gastric cancer. Clin Nutr. Epub May 13, 2017

41. Ligthart S, Marzi C, Aslibekyan S, et al. DNA methylation signatures of chronic low-grade inflammation are associated with complex diseases. Genome Biol. 2016;17(1):255.

42. Ma LN, Liu XY, Luo X,et al. Serum high-sensitivity C-reactive protein are associated with $\mathrm{HBV}$ replication, liver damage and fibrosis in patients with chronic hepatitis B. Hepatogastroenterology. 2015;62(138):368-372.
Cancer Management and Research

\section{Publish your work in this journal}

Cancer Management and Research is an international, peer-reviewed open access journal focusing on cancer research and the optimal use of preventative and integrated treatment interventions to achieve improved outcomes, enhanced survival and quality of life for the cancer patient. The manuscript management system is completely online and includes

\section{Dovepress}

a very quick and fair peer-review system, which is all easy to use. Visit http://www.dovepress.com/testimonials.php to read real quotes from published authors. 